\title{
Zukunftsfähige Organisationsstrukturen gestalten
}

\author{
Optionen für Hochschulen
}

\section{Einleitung}

Hochschulen als Organisation zu beschreiben, stellt an sich schon eine Herausforderung dar. Hochschulen sind anders als gewinnorientierte Unternehmen und unterscheiden sich ebenfalls von der öffentlichen Verwaltung. Derzeitige Anforderungen an Hochschulen lassen sich inhaltlich mit Studium und Lehre, Forschung und Wissenschaftlichkeit beschreiben. Weitere Anforderungen sind Rechtmäßigkeit im staatlichen Sinne und im Zuge der Reformen Effektivität und Effizienz, Transparenz, Partizipation, verstärkte Demokratisierung, OutputOrientierung und ein Wandel des Führungsstils (Becker/Stang 2020).

Blickt man auf die Hochschulentwicklung nun aus der Perspektive des Lernens, so spielen weitere Faktoren wie der Shift from Teaching to Learning (Barr/ Tagg 1995) eine große Rolle im Kontext von Veränderungsanforderungen. Im Zusammenhang der leistungsbezogenen Mittelzuweisung gilt es auch zu bedenken, dass alle Aufgaben der Hochschule (Forschung, Lehre, Third Mission, etc.) von gleicher Relevanz sind. Dies stellt eine Anforderung an die Hochschulen in ihren Verhandlungen mit den Ländern dar, da die leistungsbezogenen Mittel oft aus den Erfolgen in der Forschung resultieren. Das Projekt Lernwelt Hochschule zeigt, dass die Bedarfe der Studierenden eine wichtige Rolle spielen und daher deren Beteiligung eine hohe Bedeutung bei der Gestaltung von Hochschulentwicklung zukommt. Hinzu kommt, dass die Hochschulen die Qualität ihrer Lehre über Schulungen der Lehrenden und das Qualitätsmanagement selbst steuern können. Auf der Ebene der Organisation bedeutet dies, die strukturellen und kulturellen Rahmenbedingungen zu schaffen (Brahm et al. 2016), welche eine studierendenorientierte Hochschulentwicklung fördern. Eine der Herausforderungen dabei ist, dass bislang kaum Hochschulstrategien vorhanden sind, die das Lernen fokussieren. Als Good Practice lassen sich hier das TOM-Modell der 
Universität Twente ${ }^{1}$ und das Qualitätsmanagement der Universität des Saarlands ${ }^{2}$ nennen (Ziegele 2019).

\section{Öffnung der Hochschulen}

Unter der Perspektive der Studierendenorientierung sieht Ziegele (2019) eine Möglichkeit, diese zu unterstützen, darin, dass das Studienangebot ausdifferenziert wird und damit individuelle Lernwege möglich werden. Durch den Erwerb von Mikrozertifikaten kann die Durchlässigkeit zwischen beruflicher und akademischer Bildung erhöht werden. In diesem Bereich sind erste Ansätze wie zum Beispiel Kontaktstudien $\mathrm{zu}$ verzeichnen. Jedoch wird die Individualisierung durch Reglementierung, wie zum Beispiel die Regelstudienzeit, erschwert. Als Good Practice ist das Studium Individuale der Leuphana Universität ${ }^{3}$ für die Studierenden zu sehen. Good Practice im Bereich der Verbindung der Bildungsbereiche ist das Angebot der Hochschule Osnabrück ${ }^{4}$ mit der grundsätzlichen Möglichkeit, Abschlüsse in Teilzeit oder berufsbegleitend zu erlangen. Um dies organisatorisch in die Lehre zu integrieren, bedarf eines Lehrmanagements, welches ebenfalls mit den Organisationsstrukturen in Passung gebracht werden muss. Dazu müssen nicht nur administrative Strukturen rund um die Zulassung und Anerkennung dieser Angebote geschaffen werden ${ }^{5}$, sondern den aus dem erhöhten Angebot resultierenden steigenden Bedarfen an Lehrenden, Räumen und Unterstützungs- und Beratungsstrukturen wie zum Beispiel Didaktikzentren, IT-Help-Desks etc. muss begegnet werden. Zudem müssen die Prozesse und Strukturen dazu geschaffen, etabliert und sinnvoll miteinander verbunden werden.

Neben den Veränderungen des New Public Managements mit der Anforderung nach stärkerer Dezentralisierung, wirkt sich diese vertikale und horizontale Öffnung der Hochschule dahingehend aus, dass die „Organisationsstrukturen innerhalb und zwischen den Hochschulen flexibler“ (Tarazon/Brückner 2016, 78) und unterschiedlichste Kooperationsformen erprobt werden (Becker/Stang

$1 \mathrm{https://www.utwente.nl/en/tom/.}$

2 https://www.uni-saarland.de/studieren/qms-lus/start/qualifikationsziele.html.

$3 \mathrm{https} / / /$ www.leuphana.de/college/bachelor/studium-individuale.html.

4 https://www.hs-osnabrueck.de/de/learningcenter/; https://www.hs-osnabrueck.de/de/studium/studienangebot/berufsbegleitend-oder-berufsintegrierend-studieren/.

5 Dies wird auf Länder- und Bundesebene gefördert: https://www.bmbf.de/de/aufstieg-durchbildung-1240.html; https://www.wettbewerb-offene-hochschulen-bmbf.de/. 
2020) sowie die „Partizipationsbereitschaft für kollektive Belange“ (Heinrich/ Kussau 2016) gestärkt wird.

\section{Flexibilisierung der individuellen Studienwege}

Aus der Perspektive der Studierendenorientierung ist es von Vorteil, die Hochschule auch nach innen zu öffnen. Das führt dazu, dass ,es gilt von der Organisation der Hochschule in Fakultäten Abschied zu nehmen und neben der fachlichen Heimat auch flexible Querstrukturen zu schaffen“ (Ziegele et al. 2019). Ebenso wichtig ist es ebenfalls, angesichts der Heterogenität der Gruppe der Studierenden, individuelle Lernwege mit individuellen Lerntempi und Lerninhalten zu gestalten. Dies gilt insbesondere für die Studieneingangsphase. Als Good Practice für diese Phase ist das Projekt mytrack - individuelles Lernen in der Studieneingangsphase $e^{6}$ der Technischen Universität Hamburg zu nennen, welches zusammen mit der Technischen Hochschule Mittelhessen und der Hochschule Fulda durchgeführt wird. Explizite Ziele sind hierbei, eine angepasste Lerngeschwindigkeit für die Lernenden anzubieten und die Heterogenität im Blick zu haben.

Der Bedarf an Individualisierung des Studiums verändert die Aufgabe der Lehrenden grundlegend. Hier stehen als Folge die Kleingruppenarbeit und eine Hinwendung zur individuellen Lernbetreuung der einzelnen Studierenden im Fokus der Aktivitäten. Dies hat für die Organisation Hochschule zur Folge, dass die Lehrenden auch in den Bereichen Supervision und Beratung geschult und dass Raum und Zeit für diese Kleingruppenarbeit geschaffen werden müssen. Auch diese Ziele können durch an den Herausforderungen orientierte Organisationsstrukturen und durch geeignete Handlungskoordination gestützt und gefördert werden, wie zum Beispiel durch den Aufbau von fach- und kompetenzorientierten Studienstrukturen, die den internen und externen Kontext der Hochschule berücksichtigen. Hierzu zählen auch, Infrastrukturen bereitzustellen, Anreize zu schaffen und eine entsprechende Finanzierung zur Verfügung zu stellen. Im nächsten Schritt gilt es, diese Strukturen zu institutionalisieren und damit in der Organisationskultur zu verankern.

Ein weiterer Schlüssel, um eine geeignete Organisationsstruktur zu entwickeln, ist die Dezentralisierung von Kompetenzen. So ist die Schaffung der Funktion der Studiendekane oder von Studiengangsbeauftragten ein Schritt in diese Richtung. Parallel dazu ist es sinnvoll, Querschnittsbereiche und -struktu-

6 https://mytrack-tuhh.de. 
ren zu schaffen, die zum Beispiel fachlich übergreifende Themen interdisziplinär verankern. Als ein Good Practice Beispiel ist das Unterstützungsangebot der Universität Konstanz ${ }^{7} \mathrm{zu}$ bewerten. Dieses Angebot spricht Lehrende an und unterstützt alle digitalen Prozesse rund um die Lehre.

\section{Anreizstrukturen für gute Lehre schaffen}

Eine Herausforderung hierbei ist die Schaffung von Anreizstrukturen, denn hier sind den Hochschulen enge Grenzen gesetzt und vielfach kommen diese Anreize von außen, durch Programme des Bundesministeriums für Bildung und Forschung (BMBF) oder Vereinbarungen von Bund und Bundesländern wie beim Qualitätspakt Lehre ${ }^{8}$ oder dem Hochschulpakt ${ }^{9}$. Hinzu kommt, dass die Möglichkeiten der Hochschulen, auf Professorinnen und Professoren und damit auf deren verfügbares reputative, ökonomische und sowie formal-mikropolitische Kapital durch die vorhandenen Steuerungsmöglichkeiten Einfluss zu nehmen, nur sehr gering ist (Hüther 2010). Zwar gibt es Preise für gute Lehre (Becker/Stang 2010, 77), aber durch Maßnahmen wie die Exzellenzinitiative und die leistungsbezogene Mittelvergabe auf der Basis von Forschungsleistungen sind die Anreize für die Lehre geringer ausgeprägt.

Um mehr als nur besonders motivierte Lehrende zur Gestaltung guter Lehre zu motivieren, kann es hilfreich sein, den Willen zur Gestaltung dieses Prozesses anzusprechen, denn die Lehre verändert sich in den Inhalten und auch in ihren Methoden. Hier eigene Vorstellungen - wenn auch in begrenztem Umfang - realisieren zu können, stellt eine Motivation dar. So berichtet Kleinmann in seiner Studie Universitätsorganisation und präsidiale Leitung von einem Interviewteilnehmer, der einen weiteren Anreiz so beschreibt, dass „,großes Lob von dem Präsidenten' (14 L1 118) neben der leistungsorientierten Mittelverteilung einen wichtigen Leistungsanreiz darstellt“ (Kleimann 2015, 658). So ist Feedback neben Zielvorgaben (Absolventinnen-/Absolventen- und Abbrecherinnen-/Abbrecherquoten) und die Möglichkeit zur Einflussnahme eine weitere Möglichkeit, Anreize zu schaffen. Weitere Maßnahmen könnten (hochschulinterne) Förderschwerpunkte oder auch monetäre Anreize für gut gestaltete Lehre sein. Aus strategischer Perspektive heraus fehlen Finanztöpfe zur Qualitätsförderung der Lehre und es liegen nur wenige Indikatoren, wie zum Beispiel die Abbrecher-/

\footnotetext{
7 https://www.uni-konstanz.de/lehren/beratung-hilfsmittel-service/digital-unterstuetzte-lehre/.

8 https://www.bmbf.de/de/qualitaetspakt-lehre-524.html.

9 https://www.bmbf.de/de/hochschulpakt-2020-506.html.
} 
Abbrecherinnequote, vor. Als Good Practice gibt Niedersachsen in seinem Hochschulgesetz (Abschnitt 4, §14b (1)) vor, dass die Studienqualitätsmittel vorrangig dafür verwendet werden sollen,

das Betreuungsverhältnis zwischen Studierenden und Lehrenden zu verbessern, zusätzliche Tutorien anzubieten und die Ausstattung der Bibliotheken sowie der Lehr- und Laborräume zu verbessern. Soweit aus den Studienqualitätsmitteln zusätzliches Lehrpersonal finanziert wird, darf es nur zu solchen Lehraufgaben verpflichtet werden, die das für die Studiengänge erforderliche Lehrangebot ergänzen oder vertiefen (NHG 2007).

Zudem gibt es in einigen Hochschulen hochschulinterne Wettbewerbe, die besonders gelungene Konzeptionen, Projekte oder Ideen belohnen. Als ein Good Practice-Beispiel ist die Ruhr Universität Bochum zu nennen, die unter anderen mit dem Projekt 5*5000 jedes Semester innovative und lernförderliche E-Learning-Projekte unterstützt ${ }^{10}$.

Ein weiterer, hochschulübergreifender Ansatz könnte ebenfalls sein, ein Lehr-Pendant zur Deutschen Forschungsgemeinschaft (DFG) zu etablieren. Eine solche Selbstverwaltungsorganisation der Hochschullehre in Deutschland könnte neben den Hochschulen ebenfalls außeruniversitären Einrichtungen, Verbänden und Marktbegleiter aus der Wirtschaft vereinen. Hierbei könnten Mittel von EU, Bund, Ländern und privaten Zuwendungen gebündelt werden. Jedoch ist es im Bereich der Lehre von Bedeutung, dass die Entwicklung von guter Lehre nie „abgeschlossen“ sein kann, anders als ein Forschungsprojekt, das ein Endergebnis „produziert“. Mit einem solchen Verbund könnte gegebenenfalls auch der zeitlichen Begrenzung von Fördermaßnahmen durch die Programmstrukturen der Ministerien begegnet werden. Zugleich würde dadurch der Transfer von Erkenntnissen und Lösungen im Bereich der Hochschullehre erheblich befördert werden - und die zurzeit individuelle Erprobung von vielen Ideen und die Erfahrungen könnten damit gebündelt für alle Hochschulen bereitgestellt werden.

\section{Neue Aufgaben und Stellen entstehen}

Eine Aufgabe, die im Wandel der Organisationsstrukturen entstehen wird, sind die veränderten Personalstrukturen. Der Wandel zur kompetenzorientierten Lehre, die stärke Heterogenität der Studierenden und der Wechsel vom Instruktions-Paradigma zum Lern-Paradigma (Stang et al. 2020a) wird einen Wandel

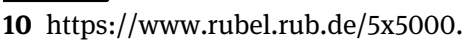


der Professionalisierung zur Folge haben. Nicht nur, dass Lehrende ihr Rollenverständnis ändern müssen - es werden veränderte professionelle Schwerpunkte entstehen, die sich vor allem auf Unterstützungsstrukturen beziehen. Hierzu zählen insbesondere die didaktische Gestaltung von Lehr-Lernprozessen, die Entwicklung und Gestaltung von Lehrmitteln oder die Konzeptionierung und Realisierung von Akkreditierungs- und Qualitätsmanagementprozessen in Bezug auf die Lehre. Weiterhin werden Lern-Coaches, aber auch Team-Coaches und Online-Coaches (Dehnbostel/Lindemann 2016, 143) benötigt werden, die soziale Kompetenzen und einen sicheren Umgang (Identitätsmanagement, Datenschutz etc. mit dem Internet vermitteln. Diese neuen Dienstleistungsstrukturen sind fachübergreifend anzusiedeln und bilden damit eine weitere horizontale Struktur in der Organisationsstruktur, die eng vernetzt mit der Bibliothek, beziehungsweise dem Medienzentrum, der IT-Abteilung und auch der Infrastrukturabteilung zusammenarbeiten muss.

\section{Möglichkeit der Learning Analytics}

Eine weitere Möglichkeit die Entwicklung zur studierendenorientierte Hochschule zu fördern ist es, ein Studienerfolgsmanagement als Teil des Qualitätsmanagements $\mathrm{zu}$ etablieren. Bisher ist ein digitales Learning-Analytics-System nur punktuell in der Hochschulwelt verbreitet. Ein Beispiel hierfür bietet die Hochschule der Medien ${ }^{11}$, welche mit LAPS (Learning Analytics für Prüfungsleistungen und Studienerfolge) Maßnahmen entwickelt, um die Studierenden, insbesondere in der Studieneingangsphase, individuell zu unterstützen. Learning Analytics adressieren, im Gegensatz zu Education Data Mining (EDM), welches die strategisch-operative und hochschulpolitische sowie ökonomische Faktoren (Erhöhung der Absolventinnen-/Absolventenquote) fokussiert, die individuelle Perspektive der Lernenden und Lehrenden und stellt die individuellen Lernprozesse in den Vordergrund (Keber 2019). Diese Analysen erlauben es den Hochschulen, typische Studienverlaufsmuster zu erfassen und die Curricula passend dazu zu entwickeln und Studierende bei der Planung ihres Studiums gezielt zu beraten. Ein weiteres Good Practice-Beispiel stellt die Technische Hochschule Köln dar, die im Rahmen ihres Qualitätsmanagements ein hochschulinternes Studienerfolgsmonitoring entwickelt hat und damit eine summative externe Effizienzbewertung erhält, die für das Auslesen von Trends und Effekten, aber

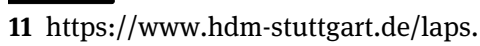


auch zur Kosten-Nutzen-Abwägung herangezogen werden kann (TeachingLearning.EU 2013).

Für die Organisation Hochschule bedeutet dies, die pädagogischen, technischen und administrativen Strukturen unter Berücksichtigung der spezifischen Situation der Hochschule in den Blick zu nehmen und die Prozesse zur Konzeption, Implementation eines nachhaltigen Betriebs aufzubauen. Auch hierbei handelt es sich um eine horizontale Ebene innerhalb der Organisationsstruktur, die alle Fachbereiche überspannt. Von großer Bedeutung ist es jedoch, Transparenz und Datenschutz zu beachten und die Teilnahme an diesen Programmen der Freiwilligkeit $\mathrm{zu}$ überlassen und verantwortungsvoll mit dem Thema Big Data umzugehen.

\section{Studierende in Entscheidungen einbinden}

Der Einbezug von Studierenden in strategische Entscheidungsbildungsprozesse in Gremien ist bei 60\% der Hochschulen verbreitet (Becker/Stang 2020, 94). Dennoch erreichen die bisherigen Partizipationsmöglichkeiten die Studierenden nicht in vollem Umfang (Aschinger 2020), sodass über neue Konzepte nachgedacht wird. Als Good Practice-Beispiel ist hier die Universität Lüneburg ${ }^{12} \mathrm{zu}$ nennen, die Studierende in Qualitätszirkeln einbindet, oder auch die Fachhochschule Potsdam ${ }^{13}$, die Studierende in die Hochschulleitung integriert. Die Hochschule Potsdam hat im Präsidium die Mitgliedschaft einer studentischen Vertreterin/eines studentischen Vertreters fest etabliert. Diese Vizepräsidentschaft ist

als Kommunikator*in und Vermittler`in zwischen Hochschulleitung und Studierendenschaft [konzipiert und] fördert die Berücksichtigung studentischer Interessen bei zentralen Entscheidungen und in Entwicklungsprozessen an der Fachhochschule (FH Potsdam 2019).

Eine weitere Möglichkeit nutzt die Universität Hildesheim ${ }^{14}$. Hier lebt die Vizepräsidentin für Lehre und Studium das Prinzip der offenen Bürotür. Ihre Sprechzeiten lauten: „bei offener Dienstzimmertür“. Des Weiteren wird zum Beispiel bei der Universität des Saarlands ${ }^{15}$ den Studierenden ermöglicht, über ein Vorschlagsformular die Verwendung der Studiengebührenkompensations-

12 https://www.leuphana.de/lehre/feedback-zu-lehre-und-studium/qualitaetszirkel.html.

13 https://www.fh-potsdam.de/informieren/organisation/hochschulleitung/.

14 https://www.uni-hildesheim.de/organe-und-gremien/praesidium/.

15 https://www.uni-saarland.de/fachrichtung/systems-engineering/studium/kompensationsmittel.html. 
mittel mitzubestimmen. Dies sind gute Möglichkeiten, die Partizipation der Studierenden $\mathrm{zu}$ erhöhen. Dies hat mehrere Vorteile für die Organisation Hochschule: mit vielfältige Formen der Partizipationsmöglichkeiten werden mehr und damit unterschiedliche Studierende erreicht, die Leitungsebene erhält wertvolle Impulse für die strategische Entwicklung und dadurch, dass es studentische Inputs sind, wird die Beteiligung und die Akzeptanz dieser Gruppe höher sein, als wenn Entscheidungen „von oben“ auferlegt erscheinen. Für die Organisationsstrukturen bedeutet dies, Kommunikationshürden abzubauen, weniger Hierarchie zu leben und kommunikative Zugänglichkeit/Offenheit zu zeigen, um diesen Bottom-up-Prozess zu stärken.

\section{Infrastruktur- und IT-Abteilungen als horizontale Struktur in der Organisation Hochschule}

Eine weitere Organisationsstruktur, welche die Entwicklung zur studierendenorientierten Hochschule unterstützt, ist die Infrastrukturabteilung, da sie die physischen Räume bereitstellt. 55 Prozent der Hochschulen haben Anschaffungen für die Ausstattungen zur Flexibilisierung der Lehr- und Lernräume bereits getätigt (Becker/Stang 2020, 102-103). Doch es fehlt an Gesamtkonzepten zur räumlichen Nutzung der Hochschule und es mangelt an systematisierten Überblicken über die räumliche Nutzungssituation (Stang et al. 2020b) und an einer durchgängigen Evaluation der Nutzung der Lehr- und Lernräume. Diese Maßnahmen würden die Auslastung und Passung zwischen den Lehr-Lernsettings und den zugeordneten Räumen verbessern und damit zum einen die angespannte Raumsituation entlasten und zum anderen die Lehr- und Lernziele unterstützen. Als Good Practice-Beispiel zeigt die SRH Hochschule Heidelberg ${ }^{16}$ mit dem CORE-Prinzip die Ausrichtung der (Innen-)Architektur an didaktische Konzepte (Ninnemann et al. 2020).

Im Zuge der Digitalisierung gilt es hierbei zu beachten, dass einerseits der physische Raum an Bedeutung gewinnt und andererseits auch die digitale Ausstattung der Räume aufgrund der kürzer werdenden technischen Innovationszyklen und den dementsprechenden Erneuerungszyklen sowie die steigende Komplexität der Medientechnik bedacht werden muss. Auch im Bereich der ITAbteilung ist eine kontinuierliche Evaluation sinnvoll. Hinzu kommen Faktoren wie die kontinuierliche Weiterbildung des technischen Personals sowie die Schulung der Nutzenden (Lehrende und Studierende) der Technik (Pirr 2017).

16 https://www.hochschule-heidelberg.de/de/core-prinzip/lernraum-campus/. 
Dies bedeutet auch, dass IT- und Infrastrukturabteilungen eng miteinander verbunden werden müssen, um eine gute Ausnutzung und Passung der Möglichkeiten zu erreichen.

\section{Fazit: Agile Strukturen als Lösung}

Betrachtet man die Themen der Hochschulentwicklung unter dem Aspekt der Organisationsstrukturen so wird klar, dass hier Anpassungen von Nöten sind. Im Bereich des Lehrmanagements bedarf es einer inneren Öffnung der Hochschulen im Sinne der curricularen Entwicklung zur Flexibilisierung und Individualisierung der Studienwege. Um die Lehrqualität zu verbessern werden Schulungen der Lehrenden in fachübergreifenden Kompetenzen wie Supervision und anderen Beratungskompetenzen erforderlich werden. Parallel dazu müssen neue Anreize geschaffen werden, welche die Lehrenden motivieren, ihre eigene Lehre weiterzuentwickeln.

Hinzu kommt die Verbesserung der Partizipationsmöglichkeiten der Studierenden in den Entscheidungsprozessen, denn sie bilden die größte Gruppe der Hochschulangehörigen und eine $\mathrm{zu}$ geringe Beteiligung (Unterrepräsentation) kann zu Legitimationsproblemen führen, zumal Studierende wertvolle Beiträge zur Entwicklung der Lehrqualität, Einrichtung und/oder Weiterentwicklung des Studien- und Beratungsangebotes sowie auch bei der Ausstattung der Hochschule einbringen können.

Weiterhin gilt es, interdisziplinäre Teams in der Organisation aufzubauen, die zum einen spezialisiert genug sind, ihre Kernaufgabe zu erfüllen, zum anderen auch generalisiert genug sind, um an den Schnittstellen mit anderen Abteilungen Themen erfassen und in ihre fachliche Perspektive transparent machen zu können. Dazu bedarf es der kontinuierlichen Aus- und Weiterbildung des technischen und wissenschaftlichen Personals. Eine weitere Möglichkeit dies zu fördern ist es, den organisationsweiten Austausch zu verbessern und damit einerseits unter anderem Parallelstrukturen, doppelte Aufwände etc. zu verringern und anderseits auch die Wissenschaftlichkeit und die Qualität der Lehre voranzutreiben.

Um die Qualität der Lehre weiterzuentwickeln könnten sich Wettbewerbe und weitere Anreizstrukturen als hilfreich erweisen, ebenso die Einführung von Learning-Analytics-Systemen, welche geeignet sind, die Effekte von Lehre nachzuverfolgen und Nachsteuerungen vorzunehmen können.

Insgesamt werden neue Querschnittsfunktionen als horizontale Verbindungen in der Organisationsstruktur entstehen. Diese werden überfachlich in den 
Bereichen der Beratungs- und Unterstützungsstrukturen angesiedelt sein, sollten allerdings ebenfalls eng an die Entscheidungs- und Entwicklungspositionen der Hochschule gekoppelt sein, da sie fachübergreifend tätig sind und somit eine starke Möglichkeit haben, Informationen in die Organisation zu tragen. Dies ist ein Schritt in Richtung Dezentralisierung, der dazu beiträgt agile und flexible Organisationsstrukturen zu entwickeln, die zukunftssicher und schnell Anpassungen an kommende Herausforderungen ermöglichen.

Die daraus entstehende hybride Organisation mit durchlässigen Abteilungsgrenzen kann situativ auf Besonderheiten reagieren und Effektivitätsvorteile nutzen. Es kann eine innovative Struktur mit flachen und anpassungsfähigen Hierarchien entstehen, welche - wenn strategisch gut geplant - die Komplexität verringert. Ein weiterer Vorteil ist darin zu sehen, dass die Interessenorganisationen (Lengfeld 2013) Lehre und Forschung mit der Arbeitsorganisation Verwaltung besser verzahnt agieren und somit ein effektiveres und effizienteres System entsteht. Um das zu erreichen, ist es jedoch notwendig, der Unsicherheit, welche Veränderungsprozesse begleitet, zu begegnen. Nachdem die strategische und organisationale Ausrichtung festgelegt ist, gilt es, die bisherigen Verhaltensmuster zu erkennen und $\mathrm{zu}$ analysieren, die zugrundeliegenden Werte, Normen, Standards der Institution Hochschule als Wertgefüge mit zum Beispiel Modi der Handlungskoordination zu verändern, um eine Innovationskultur nachhaltig zu verankern.

\section{Literatur}

Aschinger, F. (2020): Konzeption und Management der Lernwelt Hochschule. In: A. Becker; R. Stang (Hrsg.): Lernwelt Hochschule. Dimensionen eines Bildungsbereichs im Umbruch. Berlin; Boston: De Gruyter Saur, 123-150.

Barr, R.; Tagg, J. (1995): From Teaching to Learning. A New Paradigm for Undergraduate Education. Change 27/6, 12-26.

Becker, A.; Stang, R. (Hrsg.) (2020): Lernwelt Hochschule im Aufbruch. Zentrale Ergebnisse einer Befragung. In: A. Becker; R. Stang (Hrsg.): Lernwelt Hochschule. Dimensionen eines Bildungsbereichs im Umbruch. Berlin; Boston: De Gruyter Saur, 71-122.

Brahm, T.; Jenert, T.; Euler, D. (2016): Pädagogische Hochschulentwicklung als Motor für die Qualitätsentwicklung von Studium und Lehre. In: T. Brahm; T. Jenert; D. Euler (Hrsg.): Pädagogische Hochschulentwicklung. Von der Programmatik zur Implementierung. Wiesbaden: Springer VS, 19-36.

Dehnbostl, P.; Lindemann, H. J. (2016): Internationalisierung der Berufsbildung. In: M. Schönebeck; A. Pellert (Hrsg.): Von der Kutsche zur Cloud. Globale Bildung sucht neue Wege. Wiesbaden: Springer VS, 125-150.

FH Potsdam (2019): Hochschulleitung. https://www.fh-potsdam.de/informieren/organisation/ hochschulleitung/. 
Heinrich M.; Kussau, J. (2016): Finanzierungsformen, Zielvereinbarung, New Public Management und Globalbudgets. In: H. Altrichter; K. Maag Merki (Hrsg.): Handbuch Neue Steuerung im Schulsystem. Educational Governance. Wiesbaden: Springer VS, 183-207.

Hüther, O. (2010): Von der Kollegialität zur Hierarchie? Eine Analyse des New Managerialism in den Landeshochschulgesetzen. Wiesbaden: VS Verlag.

Keber, T. O. (2019): Learning Analytics. Datenschutzrechtliche und ethische Überlegungen zu studienleistungsbezogenen Datenanalysen an Hochschulen. JurPC-Web-Dok. 0097/2019. https://www.jurpc.de/jurpc/show?id=20190097\#fn0.

Kleimann, B. (2015): Universitätsorganisation und präsidiale Leitung. Führungspraktiken in einer multiplen Hybridorganisation. Wiesbaden: Springer.

Lengfeld H. (2013): Interessenorganisation. In: S. Mau; N. Schöneck (Hrsg.): Handwörterbuch zur Gesellschaft Deutschlands. Wiesbaden: Springer VS, 422-435.

Mintzberg, H. (1983): Structures in Fives. Designing Effective Organizations. Englewood Cliff: Prentice Hall.

NHG (2007): Niedersächsisches Hochschulgesetz (NHG) in der Fassung vom 26. Februar 2007 (Nds.GVBI. Nr.5/2007, 69). http://www.schure.de/22210/nhg.htm.

Ninnemann, K.; Rózsa, J.; Sutter, C. (2020): Zur Relevanz der Verknüpfung von Lernen, Raum und Organisation. Lessons Learned beim hochschulweiten Paradigmenwechsel vom Lehren zum Lernen an der SRH Hochschule Heidelberg. In: R. Stang; A. Becker (Hrsg.): Zukunft Lernwelt Hochschule. Perspektiven und Optionen für eine Neuausrichtung. Berlin; Boston: De Gruyter Saur, 179-190.

Pirr, U. (2017): Die räumliche Komponente digitaler Lehre. Ein Erfahrungsbericht. Die Hochschule. Journal für Wissenschaft und Bildung 26/2, 51-58.

Stang, R., Gläser, C.; Weckmann, H.-D.; Franke, F. (2020a): Zur Situation der Lernwelt Hochschule. Grundlagen des Projektes Lernwelt Hochschule. In: A. Becker; R. Stang (Hrsg.): Lernwelt Hochschule: Dimensionen eines Bildungsbereiches im Umbruch. Berlin; Boston: De Gruyter Saur, 9-18.

Stang, R.; Becker, A.; Franke, F.; Gläser, C.; Petschenka,, A.; Weckmann, H.-D.; Zulauf, B. (2020b): Herausforderungen Lernwelt Hochschule. Perspektiven für eine zukünftige Gestaltung. In: A. Becker; R. Stang (Hrsg.): Lernwelt Hochschule: Dimensionen eines Bildungsbereiches im Umbruch. Berlin; Boston: De Gruyter Saur, 182-210.

Tarazona M.; Brückner Y. (2016): Finanzierungsformen, Zielvereinbarung, New Public Management und Globalbudgets. In: H. Altrichter; K. Maag Merki (Hrsg.): Handbuch Neue Steuerung im Schulsystem. Educational Governance. Wiesbaden: Springer VS, 75-105.

Teaching-Learning.EU (2013): Innovationen für die Zukunft der Lehre in den Ingenieurswissenschaften. https://www.th-koeln.de/mam/downloads/deutsch/hochschule/fakultaeten/ informatik_und_ingenieurwissenschaften/mentoring/publikationen_2013_teaching.pdf.

Weick, K. E. (2009): Bildungsorganisationen als lose gekoppelte Systeme. In: S. Koch; M. Schemmann (Hrsg.): Neo-Institutionalismus in der Erziehungswissenschaft. Wiesbaden: VS Verlag für Sozialwissenschaften, 85-109.

Ziegele, F. (2019): Studierendenzentrierung durch Hochschulentwicklung. Ansatzpunkte und Potenziale. Vortrag auf der Konferenz „Zukunft Lernwelt Hochschule“ vom 27.3-28.3.2019 in Heilbronn. http://zukunftlernwelthochschule.de/wp-content/uploads/ZLH-Pr\%C3\% A4sentation-Ziegele-1.pdf.

Ziegele, F.; Neubert, P.; Mordhorst, L. (2019): Die Hochschule der Zukunft. Fels in der Brandung? Hochschulsport 2, 20-22. 